\title{
Rédaction d'un glossaire destiné à un manuel d'anglais médical : le cas du manuel Minimum Competence in Medical English
}

Josiane Hay

\section{OpenEdition}

\section{Journals}

Édition électronique

URL : http://journals.openedition.org/asp/589

DOI : $10.4000 /$ asp.589

ISBN : 978-2-8218-0404-3

ISSN : 2108-6354

\section{Éditeur}

Groupe d'étude et de recherche en anglais de spécialité

\section{Édition imprimée}

Date de publication : 1 décembre 2007

Pagination : 179-187

ISSN : 1246-8185

\section{Référence électronique}

Josiane Hay, « Rédaction d'un glossaire destiné à un manuel d'anglais médical : le cas du manuel Minimum Competence in Medical English », ASp [En ligne], 51-52 | 2007, mis en ligne le 01 décembre 2010, consulté le 22 mars 2021. URL : http://journals.openedition.org/asp/589 ; DOI : https://doi.org/ 10.4000/asp.589

Ce document a été généré automatiquement le 22 mars 2021

Tous droits réservés 


\title{
Rédaction d'un glossaire destiné à un manuel d'anglais médical : le cas du manuel Minimum Competence in Medical English
}

\author{
Josiane Hay
}

\section{Introduction : présentation générale}

\subsection{Aire lexicale}

Minimum Competence in Medical English (Upjohn et al. 2006) n'est pas destiné à une catégorie déterminée de professionnels de la santé. C'est un manuel général et, à ce titre, il n'a pas l'ambition de couvrir l'ensemble des lexiques spécialisés du vaste domaine lexical de la santé. De ce fait, lors de la rédaction, il a fallu faire des choix thématiques et de niveaux de technicité. Il a donc été décidé de proposer un travail sur des textes tirés de documents authentiques publiés sur papier ou sur Internet, parfois légèrement modifiés pour tenir compte de l'objectif du manuel et de la cohérence du passage prélevé, de difficulté moyenne et traitant de sujets à caractère médical aussi variés que possible. Le glossaire a été élaboré à partir du contenu de l'ouvrage. Il fournit aux étudiants la traduction de la majeure partie des termes employés dans le manuel. Il ne s'agit ni d'un dictionnaire ni d'un glossaire médical classique présentant des éléments lexicaux classés par spécialité.

\subsection{Choix didactiques}

2 Le glossaire a été conçu non seulement pour remplir une fonction d'aide à la compréhension, mais aussi pour servir d'outil d'apprentissage ou d'enrichissement du lexique. Il propose à l'apprenant qui interrompt un exercice ou une lecture pour chercher le sens d'un terme inconnu de profiter de cette démarche active pour 
compléter ses connaissances lexicales : le glossaire lui offre en effet non seulement la traduction du terme recherché, mais aussi la possibilité d'aller au-delà de celle-ci. Dans cette perspective, un certain nombre de choix didactiques ont été faits.

\subsubsection{Traductions multiples}

3 Il est avéré qu'à l'exception de certains rares termes habituellement techniques et récents, il n'existe pas de correspondance parfaite entre un terme d'une langue et une traduction dans une autre langue. Chaque terme possède son aire sémantique, non superposable à celle du terme choisi pour traduction. Par ailleurs, le mot tire son sens du contexte dans lequel il est employé. Pour cette raison, les listes de traductions terme à terme sont généralement peu productives et source de nombreuses erreurs. D'une part, elles n'apportent aucune information contextuelle et d'autre part elles donnent souvent à l'apprenant l'illusion que les termes qui se font face sont parfaitement équivalents. J'ai fait l'hypothèse que les traductions multiples permettraient de lutter contre ces écueils. Chaque entrée du glossaire est donc suivie de plusieurs traductions puis, chaque fois que cela a été possible, de collocations, afin de permettre à l'apprenant de commencer à construire l'aire sémantique du terme en adresse, ce qui lui permettra, par déduction, de deviner d'autres sens dans d'autres contextes.

Exemple. injury: blessure, lésion(s), traumatisme, dommages (corporels), accident,

brain injury : traumatisme cérébral

Il est évident, à la lecture des traductions offertes, que l'aire sémantique de injury dépasse celle de blessure, et qu'il faudra choisir parmi les termes offerts selon le contexte dans lequel injury est employé. L'apprenant devra donc « essayer » les diverses traductions pour trouver celle qui convient le mieux. La lecture de la liste devrait par ailleurs permettre à l'apprenant de s'imprégner des traductions pour commencer à construire le sens du terme anglais ou pour renforcer ou compléter sa connaissance.

Exemple. Le chapitre 7 comporte le terme clearance dans l'expression pulmonary interstitial fluid clearance. Le glossaire propose: "Clearance: déblaiement, dégagement, élimination, évacuation; espace libre; autorisation, approbation, formalités de contrôle ".

5 Une connaissance du sens d'œdème pulmonaire permet de sélectionner " élimination » et d'obtenir la traduction "élimination du fluide interstitiel pulmonaire». Une deuxième lecture des traductions proposées permet de comprendre que le premier sens de clearance est celui de "dégager", donc "faire de la place» (dégager, évacuer, éliminer, etc.), d'où "espace libre». Mais le sens «éliminer» mène aussi à «élimination des difficultés (administratives) », d'où « donner une autorisation », et au processus permettant de donner cette autorisation, d'où les «formalités de contrôle ».Une lecture attentive de la liste permet donc, à partir d'un noyau central, d'élargir le sens du termepour ainsi faire face à d'autres sens liés à d'autres contextes, par exemple un panneau indiquant clearance à l'entrée d'un pont ou d'un tunnel, c'està-dire espace libre (pour passer sous le pont ou le tunnel), traduit en français par «hauteur ». Par le même processus d'extension du sens, clearance peut être compris dans d'autres contextes où il peut être traduit par enlèvement, feu vert ou visa alors qu'une simple équivalence "clearance = élimination" ne permet pas d'accéder par déduction à ces autres sens. En d'autres termes, l'apprenant construit un réseau sémantique. 
6 Les listes de traduction comportent toutefois un danger : la longueur. Une liste trop longue présente le risque de saturer la mémoire ou de ne pas permettre de suivre le fil reliant les sens. Ceci explique pourquoi, de manière générale, le glossaire ne fournit que des listes relativement modestes. S'il existe quelques exceptions, telles identify (treize traductions), celles-ci indiquent que le terme anglais possède une large aire sémantique sans équivalent en français et qu'il faut donc trouver une traduction adaptée à chaque contexte. En d'autres termes, une longue liste met en garde l'apprenant : comprendre identify exige de la réflexion - la variété des contextes dans lesquels le verbe est utilisé a pour corollaire un grand nombre de traductions possibles.

\subsubsection{Regroupement sous une même entrée des termes possédant le même lemme}

7 Les termes possédant une forme identique mais appartenant à des catégories grammaticales différentes ont été regroupés sous une même entrée. Ce choix s'appuie sur une autre hypothèse : rendre les apprenants sensibles à la souplesse de l'anglais un même terme peut être nom, adjectif, adverbe, préposition et verbe - et favoriser l'apprentissage des autres catégories grammaticales que celle recherchée puisque l'apprenant lira l'article pour y trouver le sens d'un terme précis.

\section{Les entrées}

\subsection{Choix des mots-entrées}

Le contenu du manuel a été traité à l'aide du logiciel WordSmith afin d'obtenir une analyse de fréquence. La liste obtenue contenait plus de 11000 mots. Une première étape a consisté à éliminer certains termes : les articles, les pronoms, les auxiliaires des verbes (shall, will...), les modaux, traités ailleurs dans le manuel, certaines prépositions fondamentales, les verbes conjugués (was, has gone, imagined...), les noms au pluriel,les noms très semblables dans les deux langues - généralement des termes techniques qui peuvent être considérés comme monosémiques, tels que stéthoscope - ainsi que quelques autres termes dont l'usage a semblé trop marginal. La liste finale comporte 2449 termes.

\subsection{Rédaction des entrées}

9 Toutes les entrées ont été rédigées en tenant compte des besoins des apprenants professionnels des secteurs de la santé à qui le manuel est destiné. Cette approche didactique a eu un certain nombre d'incidences.

\subsubsection{Acceptions}

10 Pour chaque entrée, un premier travail a consisté à vérifier dans le manuel les contextes dans lesquels le terme est utilisé afin de sélectionner le ou les sens relevant du domaine de la médecine et de s'assurer ainsi de leur présence dans le glossaire. Comme indiqué plus haut, j'avais envisagé de ne donner pour chaque terme que le ou les sens dans le(s)quel(s) celui-ci était employé dans le manuel puisqu'on pouvait supposer que l'apprenant consulterait le glossaire uniquement pour trouver le sens 
d'un terme rencontré dans un texte. Toutefois, dans l'optique d'un outil d'apprentissage, il m'a semblé intéressant de fournir d'autres sens qui peuvent s'avérer utiles dans le domaine considéré. Par exemple, advance dans medical advance se traduit par «progrès de la médecine ", mais il peut être utile pour un apprenant de savoir que advance peut aussi signifier, entre autres, "avance, progression, amélioration, découverte ». J'ai ainsi considéré que l'apprenant pouvait enrichir son lexique par un travail d'élargissement en spirale à partir d'un terme central (celui dont on cherche le sens au départ et qui est fourni en contexte dans le manuel). De ce fait, pour les termes répertoriés, le glossaire ne se borne pas à fournir le ou les sens illustrés par le manuel.

11 Par exemple, bear apparaît une seule fois dans le manuel dans le sens de porter (carry). J'ai choisi d'étoffer l'entrée et de présenter trois groupes sémantiques «1. porter, supporter, tolérer 2. assumer 3. mettre au monde, donner naissance ».

12 En développant cette approche, j'ai décidé de fournir quelques exemples ou collocations présentant un intérêt pour un professionnel de la santé : pour bear j'ai donc ajouté child bearing age et bearing this fact. Enfin, là où cela me semblait judicieux, j'ai proposé des exemples de verbes dits à particule. Toujours pour bear, j'ai complété par : bear out « confirmer, corroborer (des hypothèses) ».

\subsubsection{Ordre des acceptions}

Dans la plupart des dictionnaires, les acceptions partent du concret pour aller vers l'abstrait. Il n'a pas été possible de faire systématiquement de même avec le glossaire : le glossaire ne fournit pas toutes les acceptions et, en outre, les acceptions concrètes très générales sont souvent absentes du glossaire. De ce fait, les acceptions sont classées du concret à l'abstrait et du général au particulier uniquement lorsque ceci est possible. Par exemple, basis: la première acception est «base, socle » et la dernière " grandes lignes ».

\subsubsection{Catégories grammaticales}

14 Les enseignants de langue étrangère savent que l'apprenant francophone classe son lexique dans des catégories grammaticales précises puisqu'en français un changement de catégorie entraîne généralement des modifications morphologiques. En anglais, en revanche, un terme passe souvent sans difficulté d'une catégorie à l'autre.

Par exemple, round peut être un nom, un verbe, un adjectif, un adverbe ou une préposition. Le nom staff «personnel » peut devenir to staff « doter en personnel ». Il me semble utile que l'apprenant s'habitue à ne pas penser le lexique en fonction de catégories grammaticales rigides lorsque qu'aucun élément morphologique (un suffixe en -ness par exemple) ne l'y contraint. Dans ce but, les termes qui figurent dans le glossaire ont été regroupés selon leur forme (lemme), sous une entrée unique. Ainsi, abuse, nom et abuse (to) verbe se trouvent sous la même entrée. En outre, tous les termes qui apparaissent dans le manuel dans une catégorie grammaticale sont " déclinés » dans toutes les autres catégories où ils présentent la même.

Exemple. L'entrée free comporte la traduction de 1. free adjectif, 2. free adverbe et 3.

free (to) verbe.

Il m'a semblé que ce regroupement permettait à l'apprenant d'une part d'enrichir son lexique et d'autre part d'acquérir l'agilité nécessaire pour passer d'une catégorie grammaticale à l'autre, et d'améliorer ainsi sa vitesse de lecture. L'ordre de traitement 
des catégories grammaticales est le suivant : nom, adjectif, adverbe, préposition, verbe et autres. Dans ces autres catégories, seuls ont été sélectionnés les termes qui relèvent du champ lexical de la médecine.

\subsubsection{Ordre et présentation des acceptions dans les listes de traduction}

De manière générale, le lexique s'est développé du concret à l'abstrait, c'est-à-dire que les termes concrets ont été utilisés pour des notions abstraites. Ainsi screen signifie tamis, c'est-à-dire tri entre ce que l'on garde et ce que l'on laisse. Ce tamis, au sens figuré, a fourni « dépistage », par analogie. Dans le glossaire, lorsque le sens concret présente un intérêt pour le domaine de la médecine, le terme a été fourni et placé au début de la liste. Les autres sens sont séparés par des puces, les termes relevant du domaine médical au sens large sont placés à la fin de l'entrée.

Prenons l'exemple de screen. La première catégorie grammaticale est lenom. Le sens «tamis» n'est pas fourni, mais en revanche le sens concret mosquito screen "moustiquaire» est fourni puisque le terme est utilisé dans le domaine la lutte antipaludique; il est suivi de antibody screen: «recherche d'anticorps». La seconde catégorie, pour ce terme, est le verbe. Le premier groupe de sens est «masquer, cacher protéger (par une moustiquaire)», puis «sélectionner, trier, filtrer», et enfin « examiner, soumettre à un contrôle (de sécurité) ». Au terme de l'entrée, on trouve pour le domaine médical «screen for : faire subir un examen de dépistage, dépister une maladie, rechercher (anticorps)».

\subsubsection{Traductions en français}

19 Pour chaque terme, j'ai tenté de fournir un large éventail de traductions afin d'aider l'apprenant à délimiter le champ sémantique du terme anglais et ainsi à mieux accéder au sens. Le terme array, par exemple traduit par "grande quantité, gamme, assortiment, série, panoplie, éventail, ensemble ». D'autre part, l'expérience montre que les apprenants à qui l'on fournit une traduction unique, réductrice, s'en contentent souvent, même lorsque celle-ci ne convient pas. J'ai donc fait le pari que les apprenants possèdent une connaissance du français suffisamment bonne pour reconnaitre le terme le mieux adapté au contexte si on le leur fournissait. J'ai fait l'hypothèse qu'un choix de termes encouragerait la réflexion sur la traduction française la mieux appropriée. Par ailleurs, ma fréquentation personnelle des dictionnaires m'a rendue sensible aux problèmes des termes inconnus rencontrés dans le corps des articles. J'ai donc décidé, lorsque cela m'a semblé utile, d'ajouter des traductions entre parenthèses au sein des articles. Par exemple, pour illustrer le sens du verbe beat «battre», j'ai précisé «pouls» et «cœur ». Dans un souci didactique, j'ai ajouté la traduction de "pouls »: pulse, et de «cœur»: heart, dans le corps de l'entrée. En effet, il m'a semblé que l'apprenant pouvait tirer parti de ces traductions, soit pour apprendre pulse et heart, soit pour revoir ces termes, sans perdre de temps à rechercher leur traduction en anglais dans un dictionnaire qu'il ou elle n'a peut-être pas à portée de main.

\subsubsection{Noms}

20 Pour les noms, la taille du glossaire ne permet pas de traiter de manière satisfaisante la question complexe des emplois comptables et non comptables ou du nombre (certains noms s'emploient plutôt au singulier ou plutôt au pluriel, ou ont un sens différent au 
singulier et au pluriel). Or les non comptables tels information ou les noms qui peuvent être comptables ou non comptables selon le sens, tels care, posent toujours des problèmes aux apprenants. La solution retenue a donc été de fournir une indication simple (n.c. = non comptable) lorsque cela était possible. Ainsi, les termes toujours non comptables dans les sens habituels sont indiqués et, là où c'est possible, quelques emplois précis sont fournis. Lorsque la situation est trop complexe, le traitement est minimaliste : seuls quelques exemples illustrent les emplois les plus fréquents.

Exemples. Coverage nom n.c.

Improvement n.c. amélioration, développement, perfectionnement : improvement of the patient's condition: amélioration de l'état du malade; pl. improvements: aménagements (maison, etc.).

\subsubsection{Adjectifs}

Les termes placés en position adjectivale posent souvent des problèmes aux apprenants francophones. Dans un but didactique, pour entraîner l'apprenant à interpréter correctement ces termes (vrais adjectifs, participes, noms), ceux-ci, étiquetés « adjectifs », figurent sous l'entrée du nom, sont traduits et souvent illustrés.

Exemple. health 1. nom n.c. santé : good/poor health: bonne, mauvaise santé • health foods: aliments diététiques, health hazard: risque/danger pour la santé, environmental health: hygiène publique $\cdot$ occupational health: hygiène/médecine $\mathrm{du}$ travail - health resort: station thermale/climatique, ville d'eaux 2. adj. de santé, sanitaire, médical $\cdot$ health authority: administration de la santé $\cdot$ health concern: soucis, problèmes de santé $\cdot$ health record: dossier médical $\bullet$ health tourism : tourisme médical • preventive health screening : dépistage médical préventif.

\subsubsection{Les verbes}

Le traitement didactique des verbes a posé divers problèmes, en particulier en ce qui concerne la transitivité et les combinaisons « verbe + préposition » ou adverbe (verbes à particule).

\section{Transitivité}

La taille du glossaire ne permet pas de traiter la transitivité de manière satisfaisante, puisque, pour un même verbe, celle-ci peut varier selon le sens. La solution adoptée a été de fournir des exemples didactiques lorsqu'il existe des différences notables entre le français et l'anglais.

Exemple.Le verbe enter est illustré par air enters the chest cavity : l'air pénètre dans la cavité pulmonaire.

24 L'exemple montre que le verbe enter est transitif direct dans le sens de "pénétrer ». Une telle explication ne reflète pas la complexité de la transitivité du verbe enter.

\section{Verbes à particule}

De même, il n'est pas possible de présenter la complexité des verbes à particule (phrasal verbs). Pour les verbes les plus courants, run par exemple, ou get, le nombre de combinaisons est très élevé : trois pages pour run ou pour get dans le grand dictionnaire bilingue Robert et Collins Senior (2002). La sélection fournie dans le glossaire se limite donc aux verbes à particule utilisés dans le manuel, à laquelle s'ajoutent parfois, dans un souci didactique, un petit nombre d'autres combinaisons essentielles pour le domaine considéré. 
exemple, pour le verbe set, le glossaire donne divers exemples dont set a fracture "réduire une facture", set a leg in plaster "plâtrer une jambe", et deux verbes à particule : set in «apparaître, se déclarer (infection)» et set up "installer mettre en place, s'installer (médecin) ». Il est évident que l'entrée set ne reflète pas la complexité sémantique du verbe.

\subsubsection{Les exemples}

Les exemples sont soit tirés du manuel, soit écrits pour le glossaire afin d'illustrer un sens ou un problème grammatical. Ils illustrent des usages du domaine médical au sens large.

Exemples. News : a news release : communiqué de presse

Neglected: neglected disease : maladie orpheline

Adverse : adverse events following immunization: manifestations postvaccinales

indésirables

Run : bedwetting runs in the family : l'énurésie est héréditaire, etc.

\subsubsection{Les ajouts}

Après avoir relu le glossaire, j'ai ajouté quelques termes que j'avais éliminés lors du premier tri, comme may, qui m'a semblé poser des problèmes de compréhension / traduction (pouvoir, risquer de ; parfois, peut-être ; il se peut que, il est possible que, il arrive que) ou over préposition (nombreux exemples).

\subsubsection{Transcription phonétique}

Le glossaire fournit une transcription phonétique rédigée en police Times NR. La transcription correspond à un accent britannique. Il nous a semblé que deux transcriptions phonétiques (une pour l'anglais britannique, une pour l'anglais américain) alourdiraient le glossaire sans être d'un réel bénéfice pour l'apprenant. La transcription phonétique a principalement pour but de guider l'apprenant lorsqu'il se trouve seul face à son manuel. En effet, nous savons que l'anglais n'est que partiellement alphabétique: si les consonnes sont relativement stables, les voyelles, elles, varient selon qu'elles se trouvent ou non sous l'accent tonique. Or, lorsque nous apprenons un mot, nous lui attribuons une image phonique. Nos étudiants francophones, face à un terme qu'ils ne connaissent pas, le cherchent dans leur dictionnaire mental oral et, s'ils ne le trouvent pas, ils le déchiffrent selon les règles de lecture du français et l'ajoutent ainsi à leur dictionnaire mental oral. Les apprenants assimilent ainsi simultanément le sens des nouveaux termes et leur image phonique lue à la française. Par exemple, la plupart des étudiants francophones prononcent nitrogen (azote) comme s'il s'agissait du vieux mot français « nitrogène ». Lorsque le terme est fréquemment utilisé, l'image phonique se fixe et il devient très difficile d'en modifier la prononciation erronée. Il nous a semblé que la transcription phonétique présentait une solution partielle au problème. Elle offre à l'apprenant isolé une béquille lui permettant de mémoriser les nouveaux termes avec leur prononciation, en particulier en ce qui concerne les voyelles problématiques faciles à déchiffrer en alphabet phonétique. Nous sommes conscients que la transcription phonétique est insuffisante et doit être complétée par l'écoute d'enregistrements, faciles à obtenir sur internet ou sur la version CD-Rom d'un dictionnaire. Ce travail additionnel d'écoute, indispensable, 
permettra à l'apprenant de fixer une image phonique suffisamment proche de la prononciation de l'anglais pour être reconnaissable par un anglophone.

\section{Conclusion}

Pour résumer, le glossaire de Minimum Competence in Medical Englisha été conçu dans un double objectif : offrir une aide à la compréhension en fournissant des traductions et permettre à l'apprenant d'effectuer un travail indépendant d'enrichissement du lexique. Cette décision a entraîné un certain nombre de choix didactiques, parfois non orthodoxes. Ainsi, le glossaire cherche à favoriser la construction en réseau de l'aire sémantique du terme anglais, une réflexion sur la traduction et l'ouverture sur les autres catégories grammaticales que le terme recherché peut éventuellement occuper. Il offre à l'apprenant la possibilité d'étoffer sa connaissance des termes recherchés par un travail d'enrichissement du lexique en spirale, à partir de l'entrée. Il fournit un certain nombre de collocations courantes et une transcription phonétique. Il est envisagé de préparer une version ultérieure de l'ouvrage comportant une version CDRom dans laquelle l'apprenant trouvera non seulement les textes du manuel, mais aussi d'autres enregistrements lus avec une variété d'accents anglais ainsi que quelques exercices. Lorsque les droits d'auteurs le permettent, les textes du manuel seront offerts en version hypertexte.

\section{BIBLIOGRAPHIE}

Upjohn J., J. Hay, P.-E. Colle, J. Hibbert et A. Depierre. 2006. Minimum Competence in Medical English. Grenoble : EDP Sciences.

\section{Ouvrages consultés}

Baker, M. 1988. « Sub-Technical Vocabulary and the ESP Teacher: An Analysis of Some Rhetorical Items in Medical Journal Articles ». Reading in a Foreign Language 4/2, 91-105.

Cambridge Advanced Learner's Dictionary. 2005. Cambridge : Cambridge University Press.

Chung, T. M. et P. Nation. 2004. « Identifying Technical Vocabulary ». System 32, 251-63.

Collins English Dictionary. 2002. Glasgow : HarperCollins.

Fröhlich, W. D. Dictionnaire de la psychologie. 1997. Paris : Le Livre de poche.

Grand dictionnaire terminologique (Le). 2007. Office québécois de la langue française <http:// granddictionnaire.com>.

Harrap's Shorter, Dictionnaire Anglais-Français/Français-Anglais. 2000. Édimbourg : Harrap.

Kernbaum, S. (dir.). Dictionnaire de Médecine. 2001. Paris : Flammarion.

Larousse, Grand Dictionnaire English-French/Français-Anglais. 1995. Paris : Larousse.

Le Mesh Bilingue Français-Anglais. 2007. INSERM. <http://ist.inserm.fr/basismesh/mesh.html>. 
Lexique Anglais-Français. 2000. Bureau de la Terminologie. Strasbourg : Éditions du Conseil de l'Europe.

Lexique Général Anglais-Français. 4 e éd., rev. et augm. 1991. New York : Nations Unies.

Macmillan English Dictionary for Advanced Learners. 2002. Oxford : Macmillan.

Medical Dictionary, basé sur Houghton Mifflin's the American Heritage ${ }^{\circledast}$ Stedman's Medical Dictionary, $2^{\mathrm{e}}$ éd. 2007. The Free Dictionary. <http://medical-dictionary.thefreedictionary.com/>.

Meertens, R. 2004. Guide anglais français de la traduction. Paris : Chiron.

Nation, I. S. P. 2001. Learning Vocabulary in Another Language. Cambridge : Cambridge University

Press.

Organisation mondiale de la santé. Genève. 2007. <http://www.who.int/en/>.

Oxford Advanced Learner's Dictionary, $7^{\mathrm{e}}$ éd. 2005. Oxford : Oxford University Press.

Oxford Collocations Dictionary for Students of English. 2002. Oxford : Oxford University Press.

Petit Robert (Le). 2002. Paris : Dictionnaires Le Robert -VUEF.

Robert \& Collins Senior. Dictionnaire français-anglais/anglais-français. 2002. Paris : Le Robert.

\section{RÉSUMÉS}

L'auteur présente le travail de rédaction du glossaire médical qui figure en annexe du manuel Minimum Competence in Medical English (Upjohn et al. 2006). Ce glossaire a été conçu pour remplir une double fonction : celle, classique, d'aide à la compréhension et celle, moins habituelle, d'outil d'apprentissage du lexique. L'auteur examine les choix didactiques qui ont structuré l'adaptation du glossaire aux besoins des apprenants professionnels des secteurs de la santé. Le parti pris didactique a, en effet, eu des incidences sur la conception d'ensemble du glossaire, sur le choix des entrées, et notamment sur le traitement du sens, des catégories grammaticales et des exemples. La question de la transcription phonétique est également abordée.

The author examines the process of compiling the medical glossary appended to the ELT manual Minimum Competence in Medical English (Upjohn et al. 2006). The glossary is intended to fulfil two functions: a traditional one - it helps students understand the texts in the manual - and a less common one - it may be used as a vocabulary learning tool. The author reviews the didactic choices structuring the adaptation of the glossary to the needs of professional learners in the various medical fields, and the impact these choices had on the overall conception of the glossary and on the choice and processing of entries, including meaning, grammatical categories and examples. Phonetic transcription is also considered.

\section{INDEX}

Keywords : glossary, language for specific purposes, language teaching manual, medical lexis, medical translation

Mots-clés : glossaire, langue de spécialité, lexique médical, manuel d'enseignement des langues, traduction médicale 
AUTEUR

JOSIANE HAY

Josiane Hay est maître de conférences à l'Université Joseph Fourier de Grenoble. Elle a été

traductrice à l'Organisation mondiale de la santé. josiane.hay@ujf-grenoble.fr 